\title{
The dichotomy of the systemic and local cytokine responses of mucosal leishmaniasis patients in Sudan: A pilot study
}

\author{
Abdelhaleem, A.A. ${ }^{1^{*}}$, Elamin, E.M. ${ }^{2}$, Elhassan, A.M. ${ }^{3}$, Nail, A.M. ${ }^{4}$, Abdullah, N.E. ${ }^{5}$ and Mukhtar, M.M. ${ }^{3,6}$ \\ ${ }^{1}$ Medical Research Center, Jazan University, Jazan 42145, Saudi Arabia \\ ${ }^{2}$ Alzaeim Alazhari University, Faculty of Medical Laboratory Sciences, Khartoum, Sudan \\ ${ }^{3}$ Institute of Endemic Diseases, University of Khartoum, Khartoum, Sudan \\ ${ }^{4}$ Tropical Diseases Teaching Hospital, Umdurman, Sudan \\ ${ }^{5}$ Department of ORL H\&N Surgery, Faculty of Medicine, University of Khartoum, Sudan \\ ${ }^{6}$ Bioscience Research Institute, Ibn Sina University, Khartoum 79371, Sudan \\ *Corresponding author email: aabdelhaleem@hotmail.com \\ Received 26 May 2020; received in revised form 8 September 2020; accepted 9 September 2020
}

\begin{abstract}
Sudanese mucosal leishmaniasis (ML) is a rare clinical form of leishmaniasis and characterized by persistent ulcer of the oral and/or the nasal mucous membranes caused by Leishmania donovani. No data is available about the systemic and local immune responses in mucosal leishmaniasis. This study aimed to measure the systemic and the local cytokines responses of Sudanese ML patients compared to cured cutaneous leishmaniasis patients (Leishmanin skin test positive, LST+ve) and unexposed healthy controls (Leishmanin skin test negative, LST-ve). Six parasitological confirmed ML patients, 7 LST+ve, and 6 LST-ve were enrolled. Systemic Th-1 (IFN- $\gamma$ and TNF- $\alpha$ ), Th-2 (IL-10 and IL-13), Treg (TGF- $\beta 1$ ), and inflammatory cytokines IL- 6 and IL- 8 concentration were measured in the supernatant of whole blood samples following stimulation with live $L$. donovani promastigotes using ELISA. Local intralesion $I L-10, I F N-\gamma$, and $I L-13$ expression was measured using Real Time PCR. A significant high concentrations of IFN- $\gamma, \mathrm{TNF} \alpha$, IL-10, TGF $\beta$, IL- 6 , and IL- 8 were detected in the supernatant of stimulated whole blood samples of ML patients compared with the LST+ve and LST-ve controls. Using Real Time-PCR and primers for various cytokines, a significant high expression of TH2 cytokines $I L-10$ and $I L-13$ mRNA was detected in contrast to a low TH1 cytokine $I F N-\gamma$ mRNA in the mucosal lesion. There is a clear dichotomy in the cytokine response during Mucosal leishmaniasis. A significantly high TH1, inflammatory and Treg cytokines response is produced systemically, in contrast to a significant high TH2 cytokines response in the mucosal lesion.
\end{abstract}

\section{INTRODUCTION}

Sudanese Mucosal leishmaniasis (ML) is a rare clinical form of Leishmaniasis presents as ulceration of the oral and/or the nasal mucous membranes (el-Hassan et al., 1995; el-Hassan et al., 2001). Unlike mucocutaneous leishmaniasis in the new world, the mucosal lesions are restricted to the mucous membranes of the oral and the nasal cavities and fail to involve the skin (el-Hassan et al., 2001; el-Hassan et al., 1994). ML usually develops in Sudanese patients after treatment of VL. Nevertheless, sporadic cases of primary ML were observed. The lesions can present as nodular growth or open ulcers not interfering with the daily activities unless infected by bacteria (el-Hassan et al., 1994). In Sudan, ML is under diagnosed due to the painless nature of the ulcers.

Sudanese ML is caused by Leishmania donovani that causes different clinical forms including Visceral Leishmaniasis (VL), Post Kala-Azar Dermal Leishmaniasis (PKDL) and 
cutaneous Leishmaniasis (CL) (Zijlstra et al., 2001a, 2001b; Elamin et al., 2008). The host cellular immune response against leishmania parasite is responsible for the immunopathology and the outcome of the infection (Caceres-Dittmar et al., 1993; Pirmez et al., 1993; Reed et al., 1993; Ismail et al., 1999; Gama et al., 2013).

While a dominant TH2 response with significant IL-10 production has been associated with susceptibility to VL, TH1 response was associated with cure (Peruhype-Magalhaes et al., 2005). In PKDL patients, a strong systemic TH1 response with dominant IFN $\gamma$ and $\mathrm{TNF} \alpha$ production was detected while a dominant TH2 response was measures in the lesions (Ismail et al., 1999). The immune response of mucocutaneous patients in the new world is mediated by strong systemic TH1 response with significant production of inflammatory cytokines (Matos et al., 2005; Costa et al., 2012). No data is available about the systemic or the local cytokines responses of Sudanese ML patients.

\section{MATERIALS AND METHODS}

\section{Ethical approval and informed consent}

The study was approved by the ethics committee of the Institute of Endemic Disease, University of Khartoum. Informed consents were obtained from all participants. The study was performed in accordance with the ethical standards stated in the 1975 Declaration of Helsinki.

\section{Participants of the study}

Patients and controls:

Six patients presented with oral and/or nasal ulcers to Khartoum Dental and the Ear, Nose and Throat (ENT) hospitals in Khartoum were suspected of ML during clinical examination and were referred to the Immunobiology laboratory at the Institute of Endemic Diseases in Khartoum for confirmation of the diagnosis. Four patients were from Gadaref State an area endemic of
Visceral Leishmaniasis, one patient was from Port Sudan city and one patient from Kassalla city east of Gadaref State.

The control included seven cured cutaneous leishmaniasis (CL) patients, reactive to Leishmanin skin test (LST+ve), and 7 healthy controls living in VL non endemic areas nonreactive to LST ( LST -ve) were enrolled as controls.

\section{Diagnosis of Mucosal leishmaniasis (ML)}

The patients were suspected of ML based on presence of persistent mucosal ulcer, who lived or visited VL endemic area. Excision biopsies were taken for routine diagnosis under local anesthesia by qualified Dental and ENT surgeons. Tissue sections were stained with H\&E and examined for presence of leishmania amastigotes. Part of the biopsy was washed with sterile normal saline, minced in sterile petri dish and inoculated into NNN media for leishmania isolation. The cultures were incubated at $24^{\circ} \mathrm{C}$ and parasite growth was observed every 3 days for 28 days.

\section{Analysis of the systemic cytokine response}

\section{Blood samples:}

Five ml of heparinized venous blood samples were aseptically collected from each patient and control by venipuncture into a sterile tube. The collected sample was diluted 1:1 in sterile Phosphate Buffered Saline (PBS). Two ml of the diluted sample were pipetted into each of 2 wells of a sterile tissue culture plate. The first well of each sample was stimulated by adding $10^{6}$ live promastigotes of $L$. donovani (previously isolated and typed from a VL patient). The other wells were kept as unstimulated controls, the unstimulated well concentration was deducted from the stimulated one. The cultures were incubated at $37^{\circ} \mathrm{C}$ under $5 \%$ $\mathrm{CO}_{2}$ for 48 hours followed by harvesting the supernatants by centrifugation at $8000 \mathrm{~g}$ for 10 minutes at $8^{\circ} \mathrm{C}$ using refrigerated centrifuge. 
Table 1. $I L-13, I L-10$ and $I F N-\gamma$ oligonucleotides primer sequences used to measure cytokine expression in mucosal tissue biopsies

\begin{tabular}{lll}
\hline$I L-13$ & FW: & 5'-TGCCATCGAGAAGACCC-3' \\
& $\mathrm{RV}:$ & 5'-TCAGTTGAACCGTCCCT-3' \\
\hline$I L-10$ & $\mathrm{FW}:$ & 5'CAGACATCAAGGCGCAT-3' \\
& $\mathrm{RV}:$ & 5'TTCTTCACCTGCTCCACG-3' \\
\hline$I F N-\gamma$ & $\mathrm{FW}:$ & 5'-GAAGAATTGGAAAGAGGAGAGTGA-3' \\
& $\mathrm{RV}:$ & 5'-GTATTGCTTTGCGTTGGAC-3' \\
\hline
\end{tabular}

Measurement of the concentrations of the cytokines in the supernatants of Peripheral blood cultures:

IFN $\gamma$, TNF $\alpha$, IL-10, IL-6, IL-8, and TGF $\beta$ concentrations were measured using cytokine specific sandwich ELSA kits (Biolegend, USA) according to the recommendations of the supplier.

\section{Measurement of the local intralesional Cytokine mRNA}

\section{Biopsy specimens:}

Part of the biopsy was used for RNA extraction using Qiagene RNA extraction kits according to the protocol of the supplier (Qiagene, USA). The extracted mRNA was converted to cDNA using cDNA synthesis kit (Promega, USA), then used to measure the expression of IFN- $\gamma$, IL-13, and IL-10 using cytokine specific primers (Table 1), $I L-13, I L-10$, and $I F N-\gamma$ primers were designed by the Light Cycler probe Design 2.0, and Oligo-4.0-S softwares (Roche Applied Science, Germany), (tested for efficiency, specificity, and sequencing of the final amplified products before used in this study). The concentration of each gene was measured in ML lesion compared with a positive control RNA (Promega, USA). The reaction was amplified by EvaGreen qPCR mix (Solis BioDyne, Estonia) following manufactures instructions. The cycling condition were $95^{\circ} \mathrm{C}$ for 15 mints preincubation, followed by 40 cycles of $95^{\circ} \mathrm{C}$ for $20 \mathrm{~s}, 60^{\circ} \mathrm{C}$ for $20 \mathrm{~s}$ and $72^{\circ} \mathrm{C}$ for $20 \mathrm{~s}$, for all primers. The prepared plate was run on a Rotor-gene Q Real time PCR machine (Qiagene USA).

\section{Data Analysis:}

The mean, the median concentrations, SD, and the P-value were determined using ANOVA, and Mann-Whitney U tests respectively. $P$ value less than 0.05 considered significant.

\section{RESULTS}

\section{Patients and Controls}

All patients were males, age more than 40 years and had typical mucosal leishmaniasis lesions (Figure 1). Four patients were residents of Gadaref state a known VL endemic area in eastern Sudan. One patient was from Kassala and the other was from Port Sudan North of Gadaref. The duration of the ulcer ranged between 2 to 10 years (Table 2). The control included seven cured cutaneous leishmaniasis (CL) patients, reactive to Leishmanin skin test (LST+ve), and 7 healthy controls living in VL non endemic areas nonreactive to LST (LST -ve) were enrolled as controls (Table 3).

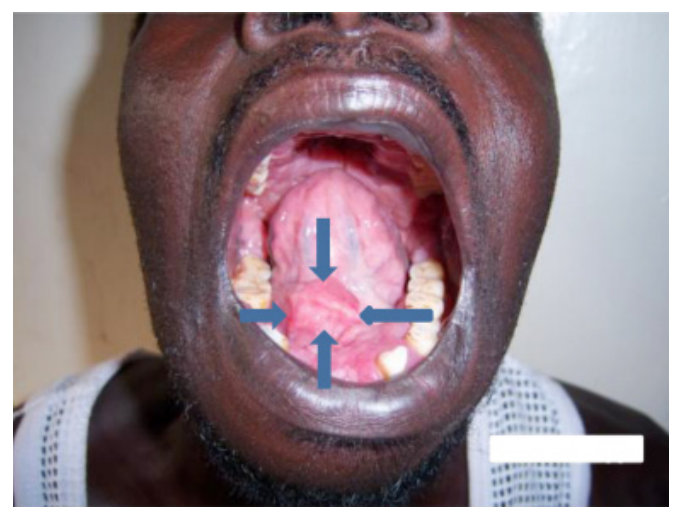

Figure 1. Patients with follicular growth mucosal Leishmaniasis lesions in the oral mucosa as indicated by arrows. 
Table 2. The number, age, gender, and duration of clinical sign of patients of the study

\begin{tabular}{ccccc}
\hline Clinical forms & Number & Range of age/ years & Gender & Duration of clinical signs \\
\hline ML & 6 & $40-55$ & Males & $2-10$ years \\
\hline
\end{tabular}

Table 3. The number, age, gender, and type of controls of the study

\begin{tabular}{lcccc}
\hline Controls & Number & Range of age/ years & Gender & Type of control \\
\hline LST positive & 7 & $20-32$ & 4 males \& 3 females & Healed \\
LST negative & 6 & $22-30$ & 2 males \& 4 females & Unexposed \\
\hline
\end{tabular}

\section{Confirmation of the diagnosis:}

Leishmania amastigotes were seen in the histological sections. Leishmania parasites were successfully cultured and typed as L. donovani using conventional PCR.

\section{Cytokine concentration of peripheral} blood samples stimulated with live leishmania donovani promastigotes:

Stimulated blood samples collected from ML patients produced a significantly high concentrations of IFN- $\gamma$, TNF $\alpha$, IL-10, TGF $\beta$, IL-6, and IL-8 compared with LST+ve and LST-ve controls $\mathrm{p}<0.05$ as shown in Figures $(2,3,4,5,6,7)$.

Measurement of $I F N-\gamma, I L-13$, and $I L-10$ mRNA in 4 ML Biopsies:

A significant high concentration of $I L-10$ mRNA was measured in the Biopsies $\mathrm{p}<0.05$, followed by $I L-13$ mRNA, compared to the low concentration of $I F N-\gamma$ mRNA (Figure 8).

\section{DISCUSSION}

Mucosal Leishmaniasis is a neglected and underdiagnosed in Sudan due to the painless nature of the disease and the treatment seeking behavior of patients. It is a rare clinical form with devastating consequence.

The number of patients enrolled in this study was small due to rare incidence of the clinical form. Our group had encountered less than 20 mucosal leishmaniasis patients during the last 20 years of active field and laboratory investigations of leishmaniasis

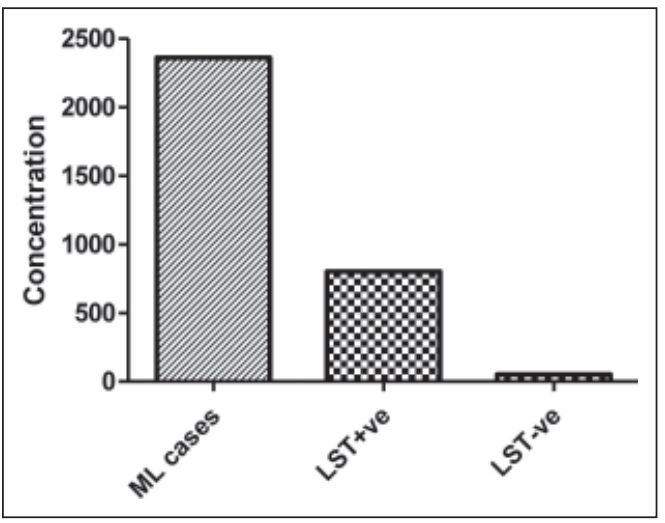

Figure 2. IFN- $\gamma$ concentration in the supernatants of whole blood of ML patients, Leishmanin skin test negative (LST -ve) and Leishmanin skin test positive (LST +ve) control following stimulation with live L. donovani promastigotes.

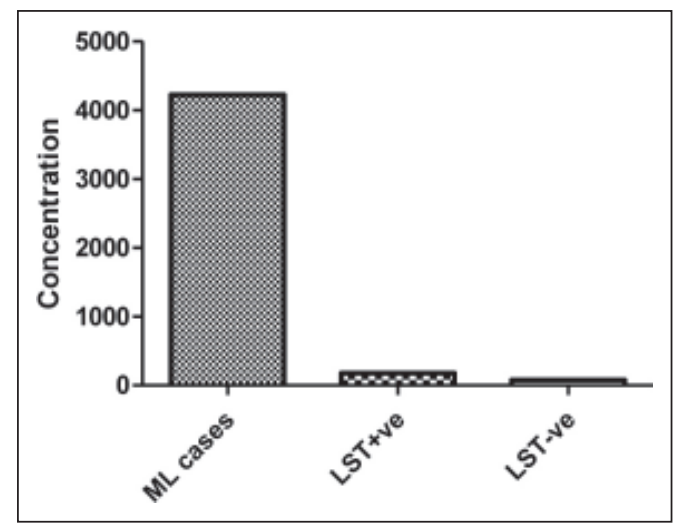

Figure 3. TNF- $\alpha$ concentration in the supernatants of whole blood of ML patients, Leishmanin skin test negative (LST -ve) and Leishmanin skin test positive (LST +ve) control following stimulation with live $L$. donovani promastigotes. 


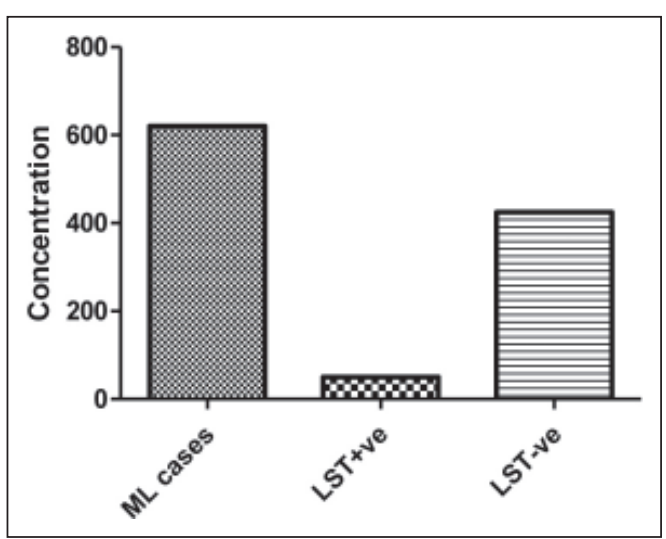

Figure 4. IL-10 concentration in the supernatants of whole blood of ML patients, Leishmanin skin test negative (LST -ve) and Leishmanin skin test positive (LST +ve) control following stimulation with live L. donovani promastigotes.

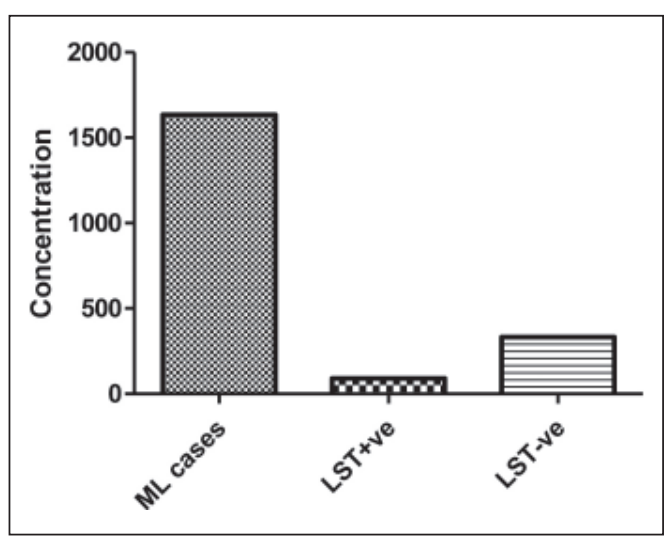

Figure 6. IL-6 concentration in the supernatants of whole blood of ML patients, Leishmanin skin test negative (LST -ve) and Leishmanin skin test positive (LST +ve) control following stimulation with live $L$. donovani promastigotes.

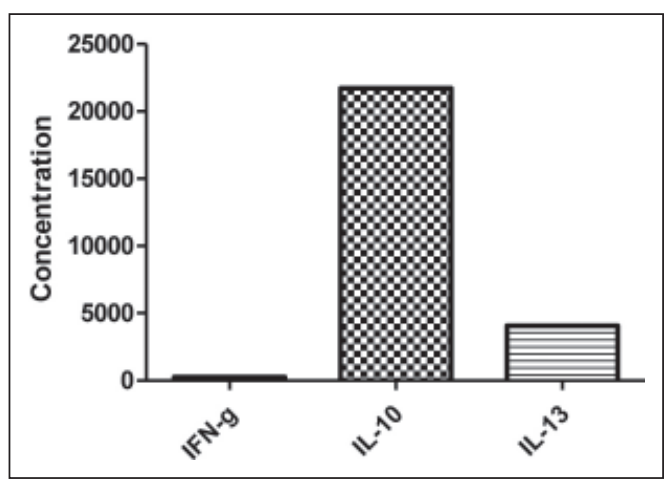

Figure 8. Median concentration of $I F N-\gamma$ ', $I L-13$; $I L-10$ expressed in lesions biopsies of ML patients.

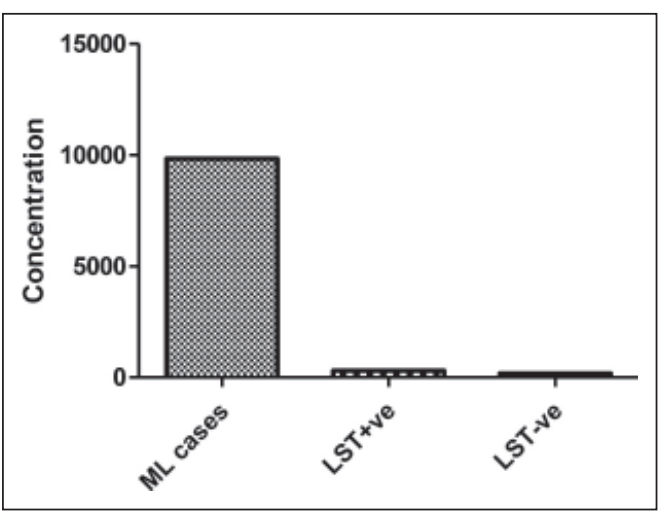

Figure 5. TGF- $\beta 1$ concentration in the supernatants of whole blood of ML patients, Leishmanin skin test negative (LST -ve) and Leishmanin skin test positive (LST +ve) control following stimulation with live $L$. donovani promastigotes.

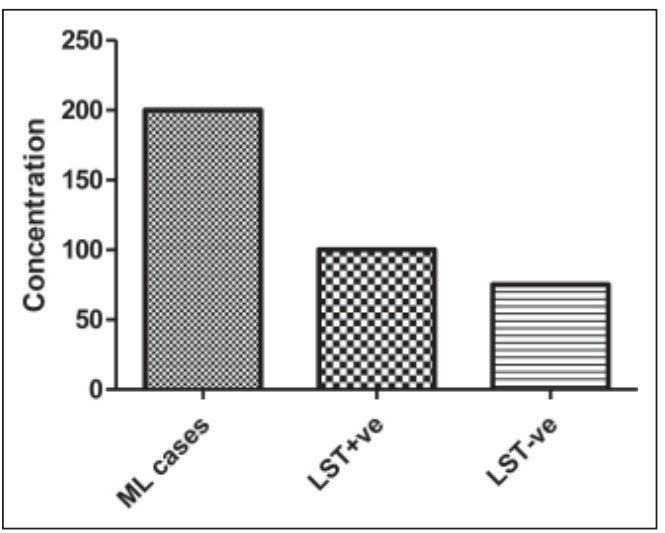

Figure 7. IL-8 concentration in the supernatants of whole blood of ML patients, Leishmanin skin test negative (LST -ve) and Leishmanin skin test positive (LST +ve) control following stimulation with live L. donovani promastigotes.

in Sudan. The enrolled patients had typical mucosal ulcers including nodular growth, and open ulcers (el-Hassan et al., 1995). The diagnosis was confirmed by detection of leishmania amastigotes in H\&E histological sections and by amplification of $L$. donovani kDNA sequence using PCR. Four patients had the common post VL mucosal leishmaniasis that developed after VL treatment, while two patients had the less frequent primary ML as observed in previous reports from Sudan (el-Hassan et al., 2001). In agreement with previous studies, males were more 
represented due to the gender treatment seeking behavior of the affected tribes where females rarely visit medical facilities for nongynecological complains (el-Hassan et al., 1995).

Several studies described the variation of the immune response during different clinical forms of Leishmaniasis. In this study, analysis of the systemic cytokine response of the enrolled ML patients showed a significant production of TH1 cytokines IFNg and TNFá, the inflammatory cytokines IL-6 and IL-8 and Treg cytokine TGF $\beta$ compared with LST+ve (cured CL patients) and LST-ve controls (unexposed). Interestingly similar cytokine responses were reported in MCL patients where a mixed TH1/TH2 response was reported with a strong TH1 dominance characterized by significant production of IFN $\gamma, \mathrm{TNF} \alpha$ and IL-8 (Caceres-Dittmar et al., 1993; Pirmez et al., 1993; Reed et al., 1993). The similarity of the cytokine response between Sudanese ML and the new world MCL is of interest since the two diseases are clinically different and caused by two different leishmania species (L. donovani and L. braziliensis). This finding indicated similarity in the pathobiology of the two parasites.

The fact that ML usually develops following successful treatment of VL is probably due to the shift of the systemic immune response of VL patients from TH2 to TH1 reflected by The strong systemic TH1 response and the production of the innate inflammatory cytokines IL-6 and IL-8 curing the parasite from the viscera while the parasites in mucosal lesions maintain a local TH2 response for their survival (Pirmez et al., 1993). This finding explains the systemic protection from relapse of ML patients to the visceral form unless the patient is immunocompromised.

In contrast to the systemic cytokine response, the intralesional cytokine expression detected a significant high production of TH2 cytokines IL-10 and IL-13 messages. The two cytokines are known to down regulate the TH1 IFN $\gamma$ and TNF- $\alpha$ responses preventing the cure of the parasite in the lesion. Similar local cytokine profiles were reported in PKDL and MCL lesions (Ismail et al., 1999; Oliveira et al., 2014).

This dichotomy of the systemic TH1 response and the intralesional TH2 response of Sudanese ML patients can explain the development of localized of ML following cure of VL leading to the survival of the parasite within the lesion.

\section{CONCLUSION}

There is a clear dichotomy in the cytokine response during Mucosal leishmaniasis. A significantly high TH1, inflammatory and Treg cytokines response is produced systemically, in contrast to a significant high TH2 cytokines response in the mucosal lesion.

Acknowledgement. The Authors would like to acknowledge all participants of the study, and staff at institute of endemic diseases university of Khartoum.

\section{Competing interests}

The author(s) declare no competing interests.

\section{Author Contribution Statement}

All authors were contributed equally in writing this manuscript.

\section{REFERENCES}

Caceres-Dittmar, G., Tapia, F.J., Sanchez, M.A., Yamamura, M., Uyemura, K., Modlen, R.L., Bloom, B.R. \& Convit, J. (1993). Determination of the cytokine profile in American cutaneous leishmaniasis using the polymerase chain reaction. Clinical and Experimental Immunology 91: 500-505.

Costa, A.S., Costa, G.C., Aquino, D.M., Mendonca, V.R., Barral, A., Barral-Netto, M. \& Caldas, A. (2012). Cytokines and visceral leishmaniasis: a comparison of plasma cytokine profiles between the clinical forms of visceral leishmaniasis. The Memorias of Oswaldo Cruz Institute 107: 735-739. 
Elamin, E.M., Guizani, I., Guerbouj, S., Gramiccia, M., El Hassan, A.M., Di Muccio, T., Taha, M.A. \& Mukhtar, M.M. (2008). Identification of Leishmania donovani as a cause of cutaneous leishmaniasis in Sudan. Transactions of The Royal Society of Tropical Medicine and Hygiene 102: 54-57.

el-Hassan, A.M., Meredith, S.E., Yagi, H.I., Khalil, E.A., Ghalib, H.W., Abbas, K., Zijlstra, E.E., Kroon, C.C., Schoone, G.J. \& Ismael, A. (1995). Sudanese mucosal leishmaniasis: epidemiology, clinical features, diagnosis, immune responses and treatment. Transactions of The Royal Society of Tropical Medicine and Hygiene 89: 647-652.

el-Hassan, A.M., Yagi, H.I., Zijlstra, E.E. \& elKadarow, A.I. (1994). Leishmaniasis of the nose caused by different species of Leishmania in the Sudan. Distinguishing features of the different clinical forms. Tropical and Geographical Medicine 46: 33-36.

el-Hassan, A.M. \& Zijlstra, E.E. (2001). Leishmaniasis in Sudan. Mucosal leishmaniasis. Transactions of The Royal Society of Tropical Medicine and Hygiene 95(1): S19-26.

Gama, M.E., Gomes, C.M., Silveira, F.T., Laurenti, M.D., Goncalves-Eda, G., da Silva, A.R. \& Corbitt, C.E. (2013). Severe visceral leishmaniasis in children: the relationship between cytokine patterns and clinical features. The Journal of the Brazilian Society of Tropical Medicine 46: 741-745.

Ismail, A., El Hassan, A.M., Kemp, K., Gasim, S., Kadaru, A.E., Moller, T. \& Kharazmi, A. (1999). Immunopathology of post kalaazar dermal leishmaniasis (PKDL): T-cell phenotypes and cytokine profile. Jourmal of Pathology 189: 615-622.
Matos, D.S., Azeredo-Coutinho, R.B. Schubach, A., Conceicao-Silva, F., Baptista, C., Moriera, J.S. \& Mendonca, S.C. (2005). Differential interferongamma production characterizes the cytokine responses to Leishmania and Mycobacterium leprae antigens in concomitant mucocutaneous leishmaniasis and lepromatous leprosy. Clinical Infectious Diseases 40: e5-12.

Oliveira, W.N., Ribeiro, L.E., Schrieffer, A. Machado, P., Carvalho, E.M. \& Bacellar, O. (2014). The role of inflammatory and anti-inflammatory cytokines in the pathogenesis of human tegumentary leishmaniasis. Cytokine 66: 127-132.

Peruhype-Magalhaes, V., Martins-Filho, O.A., Prata, A., Silva, L.A., Rabello, A., Teixeira-Carvalho, A., Figueiredo, R.M., Guimarães-Carvalho, S.F., Ferrari, T.C.A. \& Correa-Oliveira, R. (2005). Immune response in human visceral leishmaniasis: analysis of the correlation between innate immunity cytokine profile and disease outcome. Scandinavian Journal of Immunology 62: 487495.

Pirmez, C., Yamamura, M., Uyemura, K., Paes-Oliveira, M., Conceicao-Silva, F. \& Modlin, R.L. (1993). Cytokine patterns in the pathogenesis of human leishmaniasis. Journal of Clinical Investigations 91: 1390-1395.

Reed, S.G. \& Scott, P. (1993). T-cell and cytokine responses in leishmaniasis. Current Opinion in Immunology 5: 524-531.

Zijlstra, E.E. \& el-Hassan, A.M. (2001a). Leishmaniasis in Sudan. Visceral leishmaniasis. Transactions of The Royal Society of Tropical Medicine and Hygiene 95(1): S27-58.

Zijlstra, E.E. \& el-Hassan, A.M. (2001b). Leishmaniasis in Sudan. Post kala-azar dermal leishmaniasis. Transactions of The Royal Society of Tropical Medicine and Hygiene 95(1): S59-76. 